\title{
Microfilaria with neoplasm - a chance diagnosis by fine needle aspiration cytology
}

\author{
Pallavi Bhuyan, Shivangi Harankhedkar, Subrat Burma, Siva Saumendra Sahu, Sitaram \\ Mahapatra, Kaumudee Pattnaik
}

Dept. of Pathology, S. C. B. Medical College, Cuttack, Odisha, India

Correspondence: Pallavi Bhuyan, Assistant Professor. Address: Dept. of Pathology, S. C. B. Medical College, Cuttack 753007, Odisha, India. E-mail: drpallavibhuyan@gmail.com

Received: February 23, 2014

Accepted: April 30, 2014

Online Published: June 10, 2014

DOI : $10.5430 /$ crcp.v1n2p125

URL: http://dx.doi.org/10.5430/crcp.v1n2p125

\section{Abstract}

Filariasis is endemic in developing countries with highest prevalence in India. Detection of microfilariae, adult worm or egg in fine needle aspirate is very unusual despite the high incidence of this parasite. Its association with neoplasia is still rare with only a few reported cases of coexistent microfilaria with neoplasm. We report a case of a 70 year male with cervical lymphadenopathy where cytological diagnosis of Non-Hodgkin's lymphoma with chance detection of filariasis was made. This case highlights the importance of cytological smear as a useful diagnostic aid for detection of parasites in clinically unsuspected cases.

\section{Key words}

Filariasis, Fine needle aspiration cytology, Non-Hodgkin's lymphoma

\section{I ntroduction}

Filariasis is a major public health problem in tropical and subtropical countries and is an endemic problem in India, transmitted by Culex mosquito bite. Wucheria bancrofti and Brugia species (B. malayi and B. timori) are responsible for $90 \%$ and $10 \%$ respectively of infections worldwide. In endemic areas filariasis causes a spectrum of diseases including (1) asymptomatic microfilaremia, (2) recurrent lymphadenitis, (3) chronic lymphadenitis with swelling of dependent limb or scrotum (elephantiasis), and (4) tropical pulmonary eosinophilia ${ }^{[1]}$. Lymphatic filariasis has a profound detrimental effect on the economy and psycho-sexual health in societies where manual labour and subsistence agriculture are important in daily life. Cytological detection of microfilaria have been reported in different lesions like breast swelling, testis, spermatic nodules, thyroid and in tumors like Ewing's sarcoma etc. by various authors ${ }^{[2-6]}$. Hence, an early detection can help prevent the adverse sequelae.

\section{Case report}

A 70 year old male presented with an enlarged left cervical lymphnode measuring $2 \mathrm{~cm}$ diameter of one month duration. (see Figure 1). A fine needle aspiration was done from the swelling. Air dried and alcohol fixed smears were stained with Leishman stain and Haematoxylin and Eosin stain respectively. Smears were highly cellular comprising of monotonous 
population of cells with high $\mathrm{N}: \mathrm{C}$ ratio having hyperchromatic nuclei and prominent nucleoli at places. A diagnosis of Non-Hodgkin's Lymphoma (NHL) was made from the cytoaspirate (see Figure 2). Cytosmears also revealed a good number of microfilariae along with the tumor cells. Microfilariae were identified as that of Wucheria bancrofti by the presence of a hyaline sheath and nuclei extending from head to tail, the tail tip being free of nuclei. However, there were no eosinophils in the cytosmears. Subsequent peripheral smear examination revealed neither eosinophilia nor microfilaria. Histopathological study of lymph node showed NHL. Immunohistochemistry showed CD 45, CD $20+$ ve. No Microfilariae were detected in histopathology slides.

Figure 1. Lymph node swelling measuring $2 \mathrm{~cm}$ diameter in the left supraclavicular region (arrow)

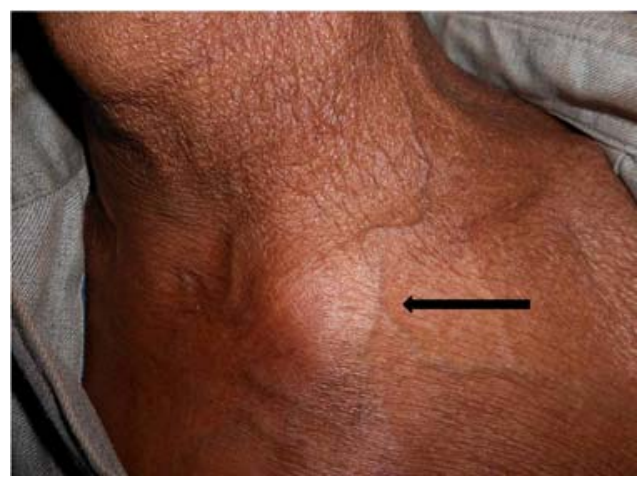

Figure 2. Cytoaspirate showing atypical lymphoid cells (arrow) along with a sheathed microfilaria (arrow head). (Leishman stain 10×)

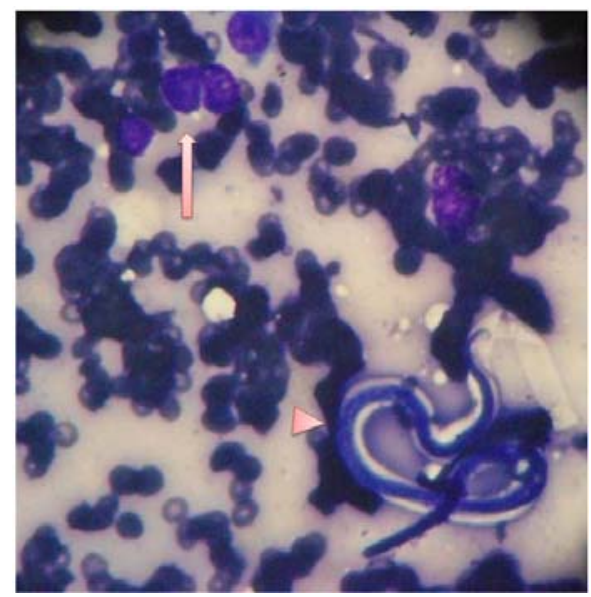

\section{Discussion}

Filarial parasites are thread like trematodes transmitted to humans by obligatory blood feeding insect vectors, Culex mosquito. Pathology mainly involves the lymphatic system and the disease manifestations are related to variations in host T-cell responses to the parasites.

Infection is initiated when female mosquitoes release infective 3rd stage larvae into the puncture site of the skin created during blood feeding. These larvae enter local lymphatic vessels, where they molt twice (4th stage larvae) to develop into sexually mature adult male and female worms. Fecund female worms release as many as 10,000 1st stage larvae (commonly called microfilariae) per day, which migrate from the lymphatics and enter blood stream. All filariae of medical significance except Loa Loa harbour rickettsia-like Wolbachia endosymbionts that are important in maintaining the reproductive capacity of adult female worms. Wolbachia, matrilineally inherited obligate intracellular bacteria, is mostly concentrated around the reproductive structure. It has been implicated in the pathogenesis of filariasis, including inflammatory responses related to filarial chemotherapy and death of the parasite. Filarial and Wolbachia antigens activate the release of proinflammatory and chemotactic cytokines which induce cellular infiltration and amplification of the inflammatory process. Toll-like receptors (TLRs) especially TLR4, appear to play a role in this process. Repeated and 
extensive exposure to filarial and Wolbachial antigens can lead to scarring and fibrosis in lymphatic filariasis and skin thickening.

Lymphatic filariasis is diagnosed by obtaining appropriate history of exposure to mosquitoes in areas where the infection is endemic, observing clinical signs of pathology and performing a variety of laboratory tests. A definitive diagnosis is made by microscopic detection of Microfilaria in the blood or occasionally from other sources such as fluid, needle aspirate, etc. Blood sample to detect Microfilaria should generally be obtained at night when peak density of parasitemia occurs. PCR-based assays detect as little as one $\mathrm{Mf} / \mathrm{ml}$ of blood. An ELISA-format antigen capture assay that detects antigen secreted by W. bancrofti adult worms has been an important diagnostic advance because this test allows documentation of active infection in the absence of microfilaremia.

Identification of a number of filarial molecules in the filarial genome project enable the organism to evade or inhibit immune defences. Brugia malayi produces: (1) Surface glycoproteins with anti-oxidant function which may protect from superoxide and free oxygen radicals, (2) Homologues of cystatins, cystein protease inhibitors, which can impair the MHC class II antigen processing pathway, (3) Serpins, serine protease inhibitors, which can inhibit neutrophil proteases, critical inflammatory mediators, and (4) Homologues of TGF- $\beta$, which can bind to mammalian TGF- $\beta$ receptors and may down regulate inflammatory responses.

Peripheral blood smear showing neither eosinophilia nor the presence of Microfilaria is consistent with the observation that in endemic areas, filariasis can exist without microfilaremia or eosinophilia ${ }^{[5]}$. Eosinophils are not mandatory to diagnose Microfilaria.

The presence of Microfilaria in cytoaspirate can be explained by the fact that larvae may be present in the vasculature and aspiration may lead to rupture of vessels resulting in hemorrhage and the release of microfilariae ${ }^{[6]}$. Cytological detection of microfilaria have been reported in lesions like breast swelling, testis and spermatic nodules, thyroid etc. by various authours ${ }^{[2,5]}$. In malignancy increased blood vasculature also causes the increase deposition of microfilaria to these sites. Coincidental detection of microfilaria with tumors have been observed in Ewing's sarcoma of bone by Ahluwalia et al. and metastatic adenocarcinomatous deposits in lymphnode by Kolte et al ${ }^{[4,6]}$.

\section{Conclusion}

Microfilariasis in association with NHL in FNAC was an incidental finding and the patient was possibly having subclinical filariasis at the time of presentation. In endemic areas, careful screening of FNAC smears helps in incidental diagnosis of co-existing pathology in clinically unsuspected cases.

\section{References}

[1] Mandell G, Douglas, Bennet's. Principles \& Practice of INFECTIOUS DISEASES. 7th ed. Churchill Livingstone. Elsevier. 2010; 2(28): 3589-3592.

[2] Gupta K, Sehgal A, Puri MM, Sidhwa HK. Microfilariae in association with other diseases. A report of 6 cases. Acta Cytol. 2002; 46: 776-778. PMid: 12146052. http://dx.doi.org/10.1159/000326996

[3] Mondal SK. Incidental detection of filarial in fine needle aspirates: a cytologic study of 14 clinically unsuspected cases at different sites. Diagn Cytopathol. 2012 Apr; 40(4): 292-6. PMid: 22431316. http://dx.doi.org/10.1002/dc.21557

[4] Kolte SS, Satarkar RN, Mane PM. Microfilaria concomitant with metastatic deposits of adenocarcinoma in lymph node fine needle aspiration cytology - A chance finding. J Cytol. 2010; 27: 78-80. PMid: 21157559. http://dx.doi.org/10.4103/0970-9371.70759

[5] Varghese TR, Raghuveer CV, Pai MR, Bansal R. Microfilaria in cytological smears: A report of six cases. Acta Cytol. 1996; 40: 290-301. http://dx.doi.org/10.1159/000333755

[6] Ahluwalia C, Choudhary M, Bajaj P. Incidental detection of Microfilaria in aspirates from Ewing's sarcoma of bone. Diagn Cytopathol. 2003; 29: 31-2. PMid: 12827713. http://dx.doi.org/10.1002/dc.10308 